\title{
Paraconsistent neurocomputing and brain signal analysis
}

\author{
Jair Minoro Abe · Helder F. S. Lopes • \\ Kazumi Nakamatsu
}

Received: 24 November 2013 / Accepted: 4 May 2014 / Published online: 8 July 2014

(c) The Author(s) 2014. This article is published with open access at Springerlink.com

\begin{abstract}
In this work we summarize some of our studies on paraconsistent artificial neural networks (PANN) applied to electroencephalography. We give attention to the following applications: probable diagnosis of Alzheimer disease and attention-deficit/hyperactivity disorder (ADHD). PANNs are well suited to tackle problems that human beings are good at solving, like prediction and pattern recognition. PANNs have been applied within several branches and among them, the medical domain for clinical diagnosis, image analysis, and interpretation signal analysis, and interpretation, and drug development. For study of ADHD, we have a result of recognition electroencephalogram standards (delta, theta, alpha, and beta waves) with a median kappa index of $80 \%$. For study of the Alzheimer disease, we have a result of clinical diagnosis possible with $80 \%$ of sensitivity, $73 \%$ of specificity, and a kappa index of $76 \%$.
\end{abstract}

Keywords Artificial neural network - Paraconsistent logics - EEG analysis - Pattern recognition - Alzheimer disease $\cdot$ Dyslexia

\footnotetext{
J. M. Abe

Graduate Program in Production Engineering,

ICET-Paulista University, R. Dr. Bacelar, 1212,

São Paulo, SP CEP 04026-002, Brazil

J. M. Abe $(\bowtie) \cdot$ H. F. S. Lopes

Institute For Advanced Studies, University of São Paulo,

São Paulo, Brazil

e-mail: jairabe@uol.com.br

H. F. S. Lopes

e-mail: helder.mobile@gmail.com

K. Nakamatsu

School of Human Science and Environment/H.S.E.,

University of Hyogo, Kobe, Japan

e-mail: nakamatu@shse.u-hyogo.ac.jp
}

\section{Introduction}

Generally speaking, artificial neural network (ANN) can be described as a computational system consisting of a set of highly interconnected processing elements, called artificial neurons, which process information as a response to external stimuli. An artificial neuron is a simplistic representation that emulates the signal integration and threshold firing behavior of biological neurons by means of mathematical structures. ANNs are well suited to tackle problems that human beings are good at solving, like prediction and pattern recognition. ANNs have been applied within several branches, among them, in the medical domain for clinical diagnosis, image analysis, and interpretation signal analysis and interpretation, and drug development.

So, ANN constitutes an interesting tool for electroencephalogram (EEG) qualitative analysis. On the other hand, in EEG analysis we are faced with imprecise, inconsistent and paracomplete data.

The EEG is a brain electric signal activity register, resultant of the space-time representation of synchronic postsynaptic potentials. The graphic registration of the sign of EEG can be interpreted as voltage flotation with mixture of rhythms, being frequently sinusoidal, ranging $1-70 \mathrm{~Hz}$ [1]. In the clinical-physiological practice, such frequencies are grouped in frequency bands as can see in Fig. 1.

EEG analysis, as well as any other measurements devices, is limited and subjected to the inherent imprecision of the several sources involved: equipment, movement of the patient, electric registers, and individual variability of physician visual analysis. Such imprecision can often include conflicting information or paracomplete data. The majority of theories and techniques available are based on classical logic and so they cannot handle adequately such set of information, at least directly. 
Fig. 1 Frequency bands clinically established and usually found in EEG
Delta: $0.1 \mathrm{~Hz}$ to $4.0 \mathrm{~Hz}$

Theta: $4.1 \mathrm{~Hz}$ to $8.0 \mathrm{~Hz}$

Alpha: $8.1 \mathrm{~Hz}$ to $12.5 \mathrm{~Hz}$

Beta: $>13 \mathrm{~Hz}$
In this paper we employ a new kind of ANN based on paraconsistent annotated evidential logic $E \tau$, which is capable of manipulating imprecise, inconsistent, and paracomplete data to make a first study of the recognition of EEG standards.

The studies about recognition of EEG standards have application in two clinical areas: attention-deficit/ hyperactivity disorder (ADHD) and Alzheimer disease (AD).

Recent researches reveal that $10 \%$ of the world population in school age suffer of learning and/or behavioral disorders caused by neurological problems, such as ADHD, dyslexia, and dyscalculia, with predictable consequences in those students insufficient performance in the school [2-7]. EEG alterations seem to be associated those disturbances. Thus, some authors have proposed that there is an increase of the delta activity in EEG in those tasks that demand a larger attention to the internal processes.

Several studies on behavioral and cognitive neurology have been conducted to characterize dementias through biological and functional markers, for instance, the EEG activity, aimed at understanding the evolution of $\mathrm{AD}$, following its progression, as well as leading toward better diagnostic criteria for early detection of cognitive impairment $[8,9]$. At present, there is no method able to determine a definitive diagnosis of dementia, where a combination of tests would be necessary to obtain a probable diagnosis [10].

Let us now make some considerations of how to apply paraconsistent artificial neural network (PANN) to analyze probable diagnosis for ADHD and AD.

\section{Background}

PANN is a new artificial neural network [11]. Its basis leans on paraconsistent annotated logic $E \tau$ [12]. Let us present it briefly.

The atomic formulas of the logic $E \tau$ are of the type $p_{(\mu, \lambda)}$, where $(\mu, \lambda) \in[0,1]^{2}$ and $[0,1]$ is the real unitary interval ( $p$ denotes a propositional variable). $p_{(\mu, \lambda)}$ can be intuitively read: "it is assumed that $p$ 's favorable evidence is $\mu$ and contrary evidence is $\lambda$ ". Thus

- $p_{(1.0,0.0)}$ can be read as a true proposition.

- $p_{(0.0,1.0)}$ can be read as a false proposition.

- $p_{(1.0,1.0)}$ can be read as an inconsistent proposition.

- $p_{(0.0,0.0)}$ can be read as a paracomplete (unknown) proposition.
Table 1 Extreme and non-extreme states

\begin{tabular}{|c|c|c|c|}
\hline Extreme states & Symbol & Non-extreme states & Symbol \\
\hline True & $\mathrm{V}$ & $\begin{array}{l}\text { Quasi-true tending } \\
\text { to inconsistent }\end{array}$ & $\mathrm{QV} \rightarrow \mathrm{T}$ \\
\hline False & $\mathrm{F}$ & $\begin{array}{l}\text { Quasi-true tending } \\
\text { to paracomplete }\end{array}$ & $\mathrm{QV} \rightarrow \perp$ \\
\hline Inconsistent & $\mathrm{T}$ & $\begin{array}{l}\text { Quasi-false tending } \\
\text { to inconsistent }\end{array}$ & $\mathrm{QF} \rightarrow \mathrm{T}$ \\
\hline \multirow[t]{5}{*}{ Paracomplete } & $\perp$ & $\begin{array}{l}\text { Quasi-false tending } \\
\text { to paracomplete }\end{array}$ & Qf $\rightarrow \perp$ \\
\hline & & $\begin{array}{l}\text { Quasi-inconsistent } \\
\text { tending to true }\end{array}$ & $\mathrm{QT} \rightarrow \mathrm{V}$ \\
\hline & & $\begin{array}{l}\text { Quasi-inconsistent } \\
\text { tending to false }\end{array}$ & $\mathrm{QT} \rightarrow \mathrm{F}$ \\
\hline & & $\begin{array}{l}\text { Quasi-paracomplete } \\
\text { tending to true }\end{array}$ & $\mathrm{Q} \perp \rightarrow \mathrm{V}$ \\
\hline & & $\begin{array}{l}\text { Quasi-paracomplete } \\
\text { tending to false }\end{array}$ & $\mathrm{Q} \perp \rightarrow \mathrm{F}$ \\
\hline
\end{tabular}

- $p_{(0.5,0.5)}$ can be read as an indefinite proposition.

We introduce the following concepts (all considerations are taken with $0 \leq \mu, \lambda \leq 1$ ):

- Uncertainty degree : $G_{\text {un }}(\mu, \lambda)=\mu+\lambda-1$

- Certainty degree : $G_{\mathrm{ce}}(\mu, \lambda)=\mu-\lambda$

Intuitively, $G_{\text {un }}(\mu, \lambda)$ show us how close (or far) the annotation constant $(\mu, \lambda)$ is from inconsistent or paracomplete state. Similarly, $G_{\mathrm{ce}}(\mu, \lambda)$ show us how close (or far) the annotation constant $(\mu, \lambda)$ is from true or false state. In this way we can manipulate the information given by the annotation constant $(\mu, \lambda)$. Note that such degrees are not metrical distance.

An order relation is defined on $[0,1]^{2}:\left(\mu_{1}, \lambda_{1}\right) \leq$ $\left(\mu_{2}, \lambda_{2}\right) \Leftrightarrow \mu_{1} \leq \mu_{2}$, and $\lambda_{2} \leq \lambda_{1}$, constituting a lattice that will be symbolized by $\tau$.

With the uncertainty and certainty degrees we can get the following 12 output states (Table 1): extreme states and nonextreme states:

Some additional control values are:

- $V_{\text {scct }}=$ maximum value of uncertainty control $=\mathrm{Ft}_{\mathrm{un}}$

- $V_{\mathrm{scc}}=$ maximum value of certainty control $=\mathrm{Ft}_{\mathrm{ce}}$

- $V_{\text {icct }}=$ minimum value of uncertainty control $=-\mathrm{Ft}_{\mathrm{un}}$

- $V_{\mathrm{icc}}=$ minimum value of certainty control $=-\mathrm{Ft}_{\mathrm{ce}}$ 


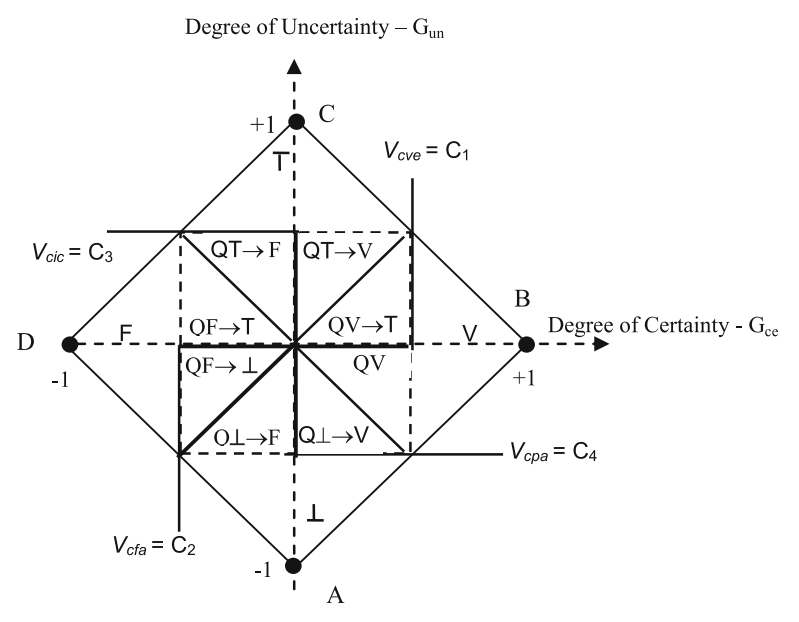

Fig. 2 Extreme and non-extreme states

Such values are determined by the knowledge engineer, depending on each application, finding the appropriate control values for each of them.

All states are represented in the next figure (Fig. 2).

\section{The main artificial neural cells}

In the PANN, the certainty degree $G_{\mathrm{ce}}$ indicates the 'measure' falsity or truth degree.

The uncertainty degree $G_{\text {un }}$ indicates the 'measure' of the inconsistency or paracompleteness. If the certainty degree in module is low or the uncertainty degree in module is high, it generates a paracompleteness.

The resulting certainty degree $G_{\mathrm{ce}}$ is obtained as follows:

- If: $V_{\mathrm{cfa}}=G_{\mathrm{ce}}=V_{\mathrm{cve}}$ or $-\mathrm{Ft}_{\mathrm{ce}}=G_{\mathrm{ce}}=\mathrm{Ft}_{\mathrm{ce}} \Rightarrow G_{\mathrm{ce}}=$ indefiniteness

- For: $V_{\text {cpa }}=G_{\text {un }}=V_{\text {cic }}$ or $-\mathrm{Ft}_{\text {un }}=G_{\text {un }}=\mathrm{Ft}_{\text {un }}$

- If: $G_{\mathrm{ce}}=V_{\mathrm{cfa}}=-\mathrm{Ft}_{\mathrm{ce}} \Rightarrow G_{\mathrm{ce}}=$ false with degree $G_{\text {un }}$

- If: $\mathrm{Ft}_{\mathrm{ce}}=V_{\mathrm{cve}}=G_{\mathrm{ce}} \Rightarrow G_{\mathrm{ce}}=$ true with degree $G_{\mathrm{un}}$

A paraconsistent artificial neural cell (PANC) is called basic PANC (Fig. 3) when given a pair $(\mu, \lambda)$ is used as input and resulting as output:

- $S_{2 \mathrm{a}}=G_{\mathrm{un}}=$ resulting uncertainty degree

- $S_{2 \mathrm{~b}}=G_{\mathrm{ce}}=$ resulting certainty degree

- $S_{1}=X=$ constant of indefiniteness.

The uncertainty degree $G_{\text {un }}$ indicates the 'measure' of the inconsistency or paracompleteness. If the certainty degree in module is low or the uncertainty degree in module is high, it generates an indefiniteness.

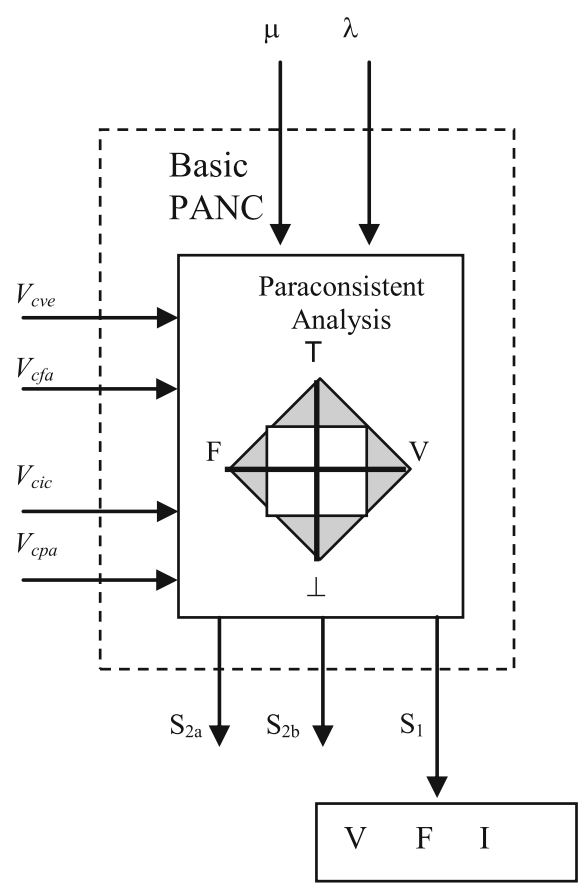

Fig. 3 Basic cell of PANN

The resulting certainty degree $G_{\mathrm{ce}}$ is obtained as follows:

- If: $V_{\mathrm{cfa}}=G_{\mathrm{ce}}=V_{\mathrm{cve}}$ or $-\mathrm{Ft}_{\mathrm{ce}}=G_{\mathrm{ce}}=\mathrm{Ft}_{\mathrm{ce}} \Rightarrow$ $G_{\mathrm{ce}}=$ indefiniteness

- For: $V_{\mathrm{cpa}}=G_{\mathrm{un}}=V_{\mathrm{cic}}$ or $-\mathrm{Ft}_{\mathrm{un}}=G_{\mathrm{un}}=\mathrm{Ft}_{\mathrm{un}}$

- If: $G_{\mathrm{ce}}=V_{\mathrm{cfa}}=-\mathrm{Ft}_{\mathrm{ce}} \Rightarrow G_{\mathrm{ce}}=$ false with degree $G_{\text {un }}$

- If: $\mathrm{Ft}_{\mathrm{ce}}=V_{\mathrm{cve}}=G_{\mathrm{ce}} \Rightarrow G_{\mathrm{ce}}=$ true with degree $G_{\mathrm{un}}$

A PANC is called basic PANC (Fig. 3) when given a pair $(\mu, \lambda)$ is used as input and resulting as output:

- $S_{2 \mathrm{a}}=G_{\mathrm{un}}=$ resulting uncertainty degree

- $S_{2 \mathrm{~b}}=G_{\mathrm{ce}}=$ resulting certainty degree

- $S_{1}=X=$ constant of Indefiniteness.

Using the concepts of basic PANC, we can obtain the family of PANC considered in this work: analytic connection (PANCac), maximization (PANCmax), and minimization (PANCmin) as described in Table 2 below:

To make easier the understanding on the implementation of the algorithms of PANC, we use a programming language Object Pascal, following logic of procedural programming in all samples.

3.1 Paraconsistent artificial neural cell of analytic connection (PANCac)

The PANCac is the principal cell of all PANN, obtaining the certainty degree $\left(G_{\mathrm{ce}}\right)$ and the uncertainty degree $\left(G_{\text {un }}\right)$ from the inputs and the tolerance factors. 
Table 2 Paraconsistent artificial neural cells

\begin{tabular}{llll}
\hline PANC & Inputs & Calculations & Output \\
\hline Analytic connection: PANCac & $\mu$ & $\lambda_{\mathrm{c}}=1-\lambda$ & If $\left|G_{\mathrm{ce}}\right|>\mathrm{Ft}_{\mathrm{ce}}$ then $S_{1}=\mu_{\mathrm{r}}$ and $S_{2}=0$ \\
& $\lambda$ & $G_{\mathrm{un}} G_{\mathrm{ce}}$, & If $\left|G_{\mathrm{un}}\right|>\mathrm{Ft}_{\mathrm{ct}}$ and $\left|G_{\mathrm{un}}\right|>\left|G_{\mathrm{ce}}\right|$ then \\
& $\mathrm{Ft}_{\mathrm{un}}$ & $\mu_{\mathrm{r}}=\left(G_{\mathrm{ce}}+1\right) / 2$ & $S_{1}=\mu_{\mathrm{r}}$ and $S_{2}=\left|G_{\mathrm{un}}\right|$ \\
& $\mathrm{Ft}_{\mathrm{un}}$ & & If not $S_{1}=1 / 2$ and $S_{2}=0$ \\
& $\mu$ & $G_{\mathrm{ce}}$ & If $\mu_{\mathrm{r}}>0.5$, then $S_{1}=\mu$ \\
Maximization: PANCmax & $\lambda$ & $\mu_{\mathrm{r}}=\left(G_{\mathrm{ce}}+1\right) / 2$ & If not $S_{1}=\lambda$ \\
& $\mu$ & $G_{\mathrm{ce}}$ & If $\mu_{\mathrm{r}}<0.5$, then $S_{1}=\mu$ \\
Minimization: PANCmin & $\lambda$ & $\mu_{\mathrm{r}}=\left(G_{\mathrm{ce}}+1\right) / 2$ & If not $S_{1}=\lambda$ \\
& $\lambda$ &
\end{tabular}

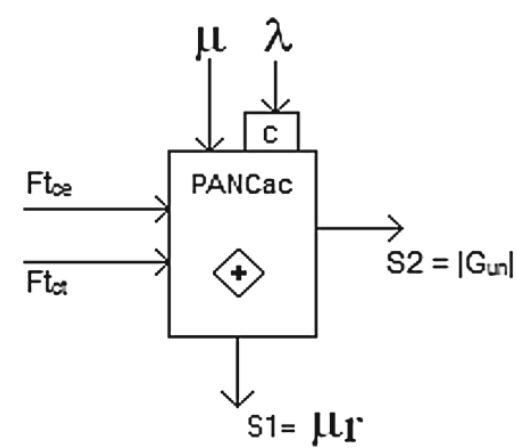

Fig. 4 Representation of PANCac

This cell is the link which allows different regions of PANN perform signal processing in distributed and through many parallel connections [11].

The different tolerance factors certainty (or contradiction) acts as inhibitors of signals, controlling the passage of signals to other regions of the PANN, according to the characteristics of the architecture developed (Fig. 4).

In Table 3, we have a sample of implementation made in Object Pascal.

\subsection{Paraconsistent artificial neural cell of maximization (PANCmax)}

The PANCmax allows selection of the maximum value among the entries.

Such cells operate as logical connectives OR between input signals. For this is made a simple analysis, through the equation of the degree of evidence (Table 4) which thus will tell which of the two input signals is of greater value, thus establishing the output signal [11] (Fig. 5).

In Table 4, we have a sample of implementation made in Object Pascal.

\subsection{Paraconsistent artificial neural cell of minimization} (PANCmin)

The PANCmin allows selection of the minimum value among the entries.
Table 3 PANCac implementation

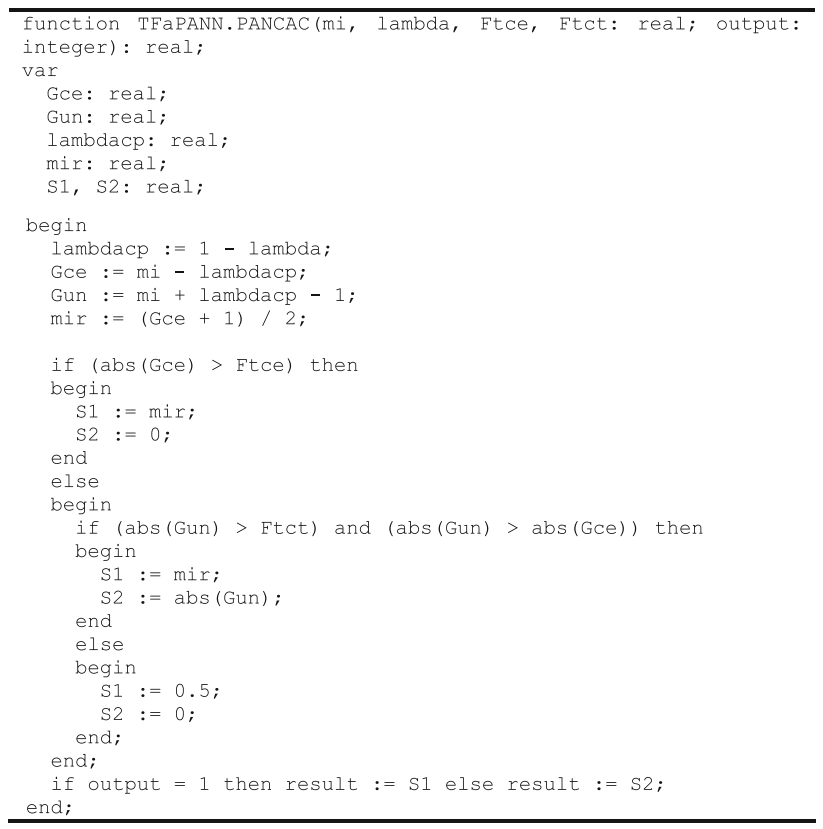

Table 4 PANCmax implementation

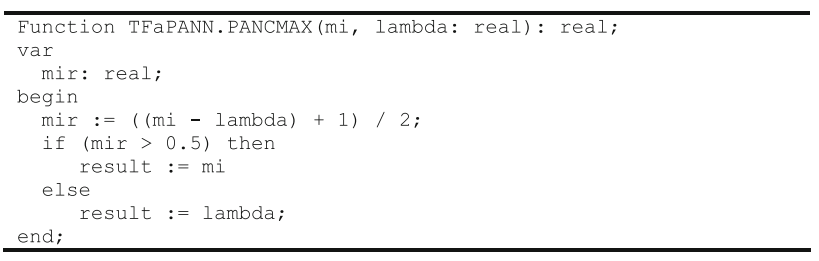

Such cells operate as logical connectives AND between input signals. For this it is made a simple analysis, through the equation of the degree of evidence (Table 5) which thus will tell which of the two input signals is of smaller value, thus establishing the output signal [11].

In Table 5, we have a sample of implementation made in Object Pascal.

3.4 Paraconsistent artificial neural unit

A PANU is characterized by the association ordered PANC, targeting a goal, such as decision making, selection, learning, or some other type of processing. 
Fig. 5 Representation of PANCmax

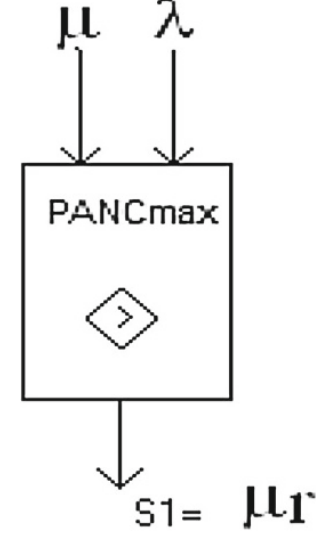

Table 5 PANCmin implementation

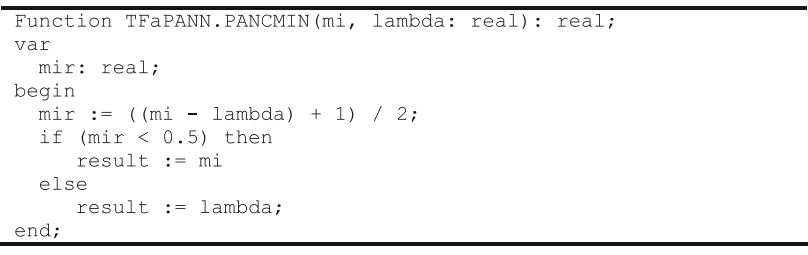

When creating a PANU, one obtains a data processing component capable of simulating the operation of a biological neuron.

\subsection{Paraconsistent artificial neural system}

Classical systems based on binary logic are difficult to process data or information from uncertain knowledge. These data are captured or received information from multiple experts usually comes in the form of evidences.

Paraconsistent artificial neural system (PANS) modules are configured and built exclusively by PANU, whose function is to provide the signal processing 'similar' to processing that occurs in the human brain.

\section{PANN for morphological analysis}

The process of morphological analysis of a wave is performed by comparing with a certain set of wave patterns (stored in the control database). A wave is associated with a vector (finite sequence of natural numbers) through digital sampling. This vector characterizes a wave pattern and is registered by PANN. Thus, new waves are compared, allowing their recognition or otherwise.

Each wave of the survey examined the EEG corresponds to a portion of $1 \mathrm{~s}$ examination. Every second of the exam contains 256 positions.

The wave that has the highest favorable evidence and lowest contrary evidence is chosen as the more similar wave to the analyzed wave.
A control database is composed by waves presenting 256 positions with perfect sinusoidal morphology, with $0.5 \mathrm{~Hz}$ of variance, so taking into account delta, theta, alpha, and beta (of $0.5-30.0 \mathrm{~Hz}$ ) wave groups.

In other words, morphological analysis checks the similarity of the passage of the examination of EEG in a reference database that represents a wave pattern.

\subsection{Data preparation}

The process of wave analysis by PANN consists previously of data capturing, adaptation of the values for screen examination, elimination of the negative cycle, and normalization of the values for PANN analysis.

As the actual EEG examination values can vary highly, in module, something $10-1,500 \mu \mathrm{V}$, we make a normalization of the values between 100 and $-100 \mu \mathrm{V}$ by a simple linear conversion, to facilitate the manipulation the data:

$x=\frac{100 \cdot a}{m}$,

where $m$ is the maximum value of the exam; $a$ is the current value of the exam; $x$ is the current normalized value.

The minimum value of the examination is taken as zero value and the remaining values are translated proportionally.

It is worth observing that the process above does not allow the loss of any wave essential characteristics for our analysis.

\subsection{The PANN architecture}

The architecture of the PANN used in decision making is based on the architecture of PANS for treatment of contradictions.

Such a system performs a treatment of the contradictions continuously if presented by the three information signal inputs, presenting as an output a resulting signal that represents a consensus among the three information. This is made by analyzing the contradiction between two signals, and by adding a third one; the output is chosen by dominant majority. The analysis is instantly carrying all processing in real time, similar to the functioning of biological neurons.

This method is used primarily for PANN (Fig. 8) to balance the data received from expert systems. After this the process uses a decision-making lattice to determine the soundness of the recognition (Table 6; Fig. 6).

A sample of morphological analysis implementation using Object Pascal is showed in Table 7.

The definition of regions of the lattice decision-making was done through double-blind trials, i.e., for each battery of tests, a validator checked the results and returned only the percentage of correct answers. After testing several different configurations, set the configuration of the lattice 
Table 6 Lattice for decision-making used in the morphological analysis (Fig. 7)

Limits of areas of lattice

\begin{tabular}{llll}
\hline True & $\mathrm{Fe}>0.61$ & $\mathrm{Ce}<0.40$ & $G_{\text {ce }}>0.22$ \\
False & $\mathrm{Fe}<0.61$ & $\mathrm{Ce}>0.40$ & $G_{\text {ce }} \leq 0.23$
\end{tabular}

$\mathrm{Ce}$ contrary evidence, $\mathrm{Fe}$ favorable evidence, $G_{\mathrm{ce}}$ certainty degree

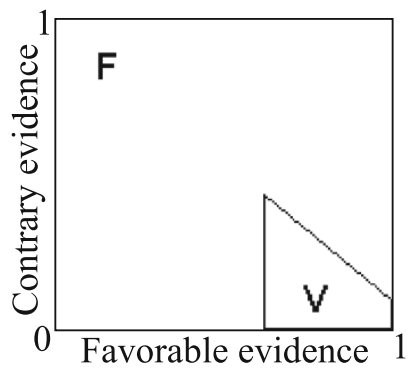

Fig. 6 Lattice for decision-making used in morphological analysis used after making PANN; $F$ logical state false (it is interpreted as wave not similar); $V$ logical state true (it is interpreted as wave similar)

Table 7 The architecture for morphological analysis implementation (Fig. 8)

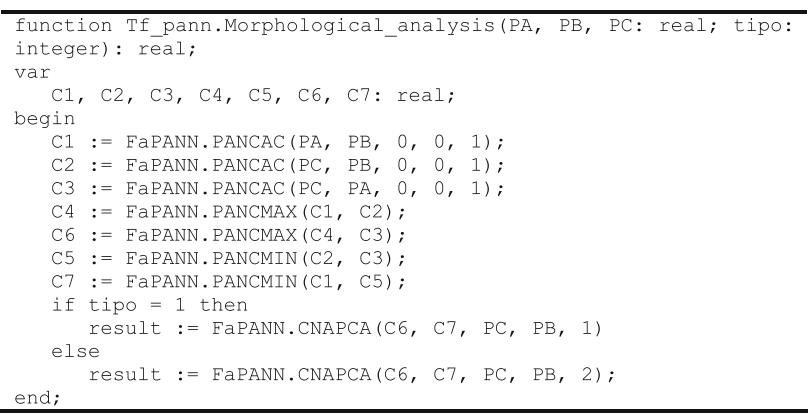

Fig. 7 Representation of PANCmin

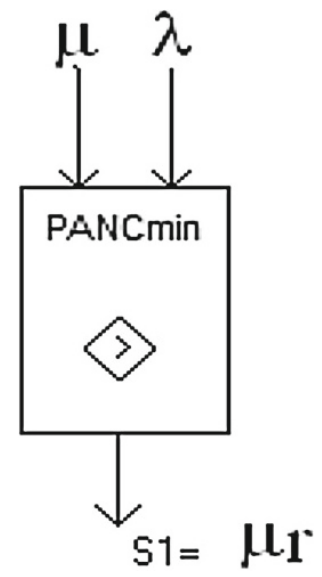

regions whose decision-making had a better percentage of success.

For an adequate PANN wave analysis, it is necessary that each input of PANN is properly calculated. These input vari-

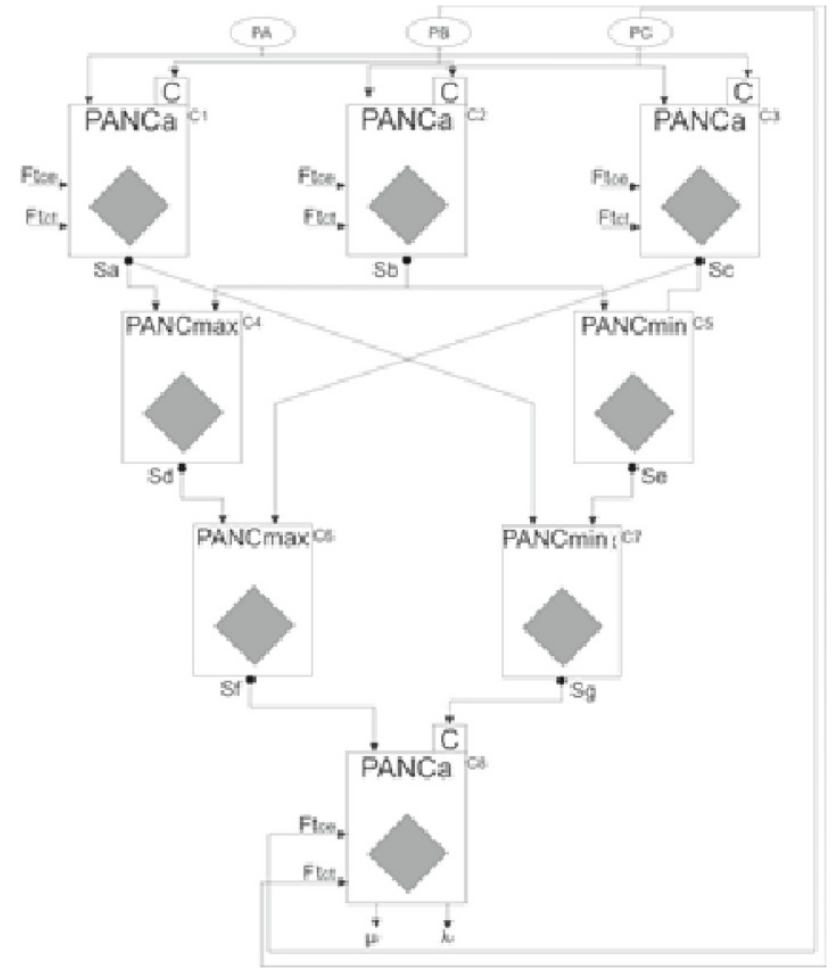

Fig. 8 The architecture for morphological analysis. Three expert systems operate: $P A$ for check the number of wave peaks; $P B$ for checking similar points, and $P C$ for checking different points The 1st layer of the architecture: $\mathrm{C} 1-\mathrm{PANC}$ which processes input data of $\mathrm{PA}$ and $\mathrm{PB}$; C2-PANC which processes input data of PB and PC; C3-PANC which processes input data of $\mathrm{PC}$ and $\mathrm{PA}$. The 2nd layer of the architecture: C4-PANC which calculates the maximum evidence value between cells $\mathrm{C} 1$ and $\mathrm{C}$; C5-PANC which calculates the minimum evidence value between cells $\mathrm{C} 2$ and $\mathrm{C} 3$; The 3rd layer of the architecture: C6-PANC which calculates the maximum evidence value between cells $\mathrm{C} 4$ and $\mathrm{C} 3$; C7-PANC which calculates the minimum evidence value between cells $\mathrm{C} 1$ and $\mathrm{C} 5$. The 4th layer of the architecture: C8 analyzes the experts $\mathrm{PA}, \mathrm{PB}$, and $\mathrm{PC}$ and gives the resulting decision value. $\mathrm{PANC} \mathrm{A}=$ paraconsistent artificial neural cell of analytic connection. PANCLs Max $=$ paraconsistent artificial neural cell of simple logic connection of max-

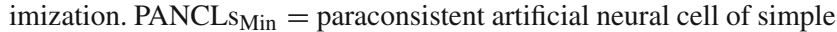
logic connection of minimization. $\mathrm{Ft}_{\mathrm{ce}}=$ certainty tolerance factor; $\mathrm{Ft}_{\mathrm{un}}=$ uncertainty tolerance factor. $S_{\mathrm{a}}=$ output of C1 cell; $S_{\mathrm{b}}=$ output of C2 cell; $S_{\mathrm{c}}=$ output of C 3 cell; $S_{\mathrm{d}}=$ output of C4 cell; $S_{\mathrm{e}}=$ output of C5 cell; $S_{\mathrm{f}}=$ output of C6 cell; $S_{\mathrm{g}}=$ output of C7 cell. C $=$ complemented value of input; $\mu_{\mathrm{r}}=$ value of output of PANN; $\lambda_{\mathrm{r}}=$ value of output of PANN

ables are called expert systems as they are specific routines for extracting information.

In analyzing EEG signals, one important aspect to take into account is the morphological aspect. To perform such a task, it is convenient to consider an expert system which analyzes the signal behavior verifying which band it belongs to (delta, theta, alpha and beta).

The method of morphological analysis has three expert systems that are responsible for feeding the inputs of PANN with information relevant to the wave being analyzed: number of peaks, similar points, and different points. 
Table 8 Checking the number of wave peaks function implementation

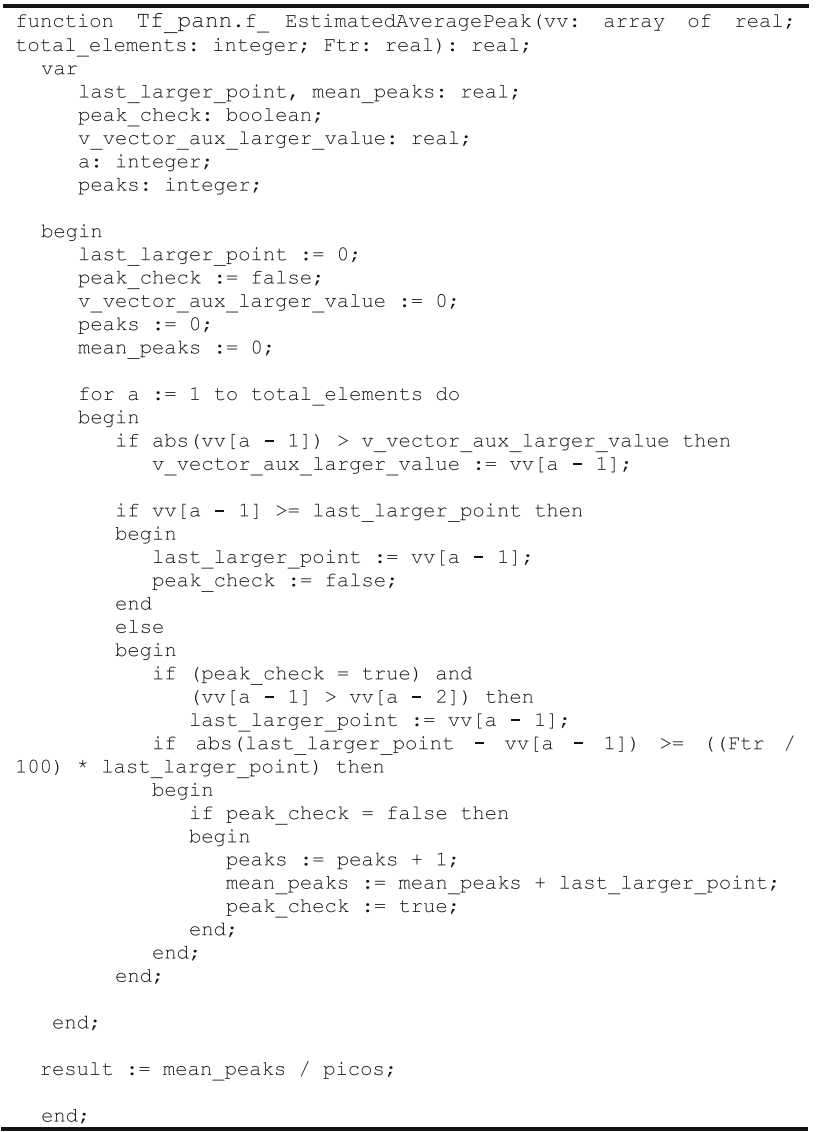

4.3 Expert system 1: checking the number of wave peaks

The aim of the expert system 1 is to compare the waves and analyze their differences regarding the number of peaks.

In practical terms, one can say that when we analyzed the wave peaks, we are analyzing the resulting frequency of wave (so well rudimentary).

It is worth remembering that, because it is biological signal, we should not work with absolute quantification due to the variability characteristic of this type of signal. Therefore, one should always take into consideration a tolerance factor.

A sample of checking the number of wave peaks function implementation using Object Pascal is show in Table 8.

$\mathrm{Se}_{1}=1-\left(\frac{(|b d-v t|)}{(b d+v t)}\right)$

where $v t$ is the number of peaks of the wave, $b d$ is the number of peaks of the wave stored in the database, $\mathrm{Se}_{1}$ is the value resulting from the calculation.
Table 9 Checking similar points function implementation

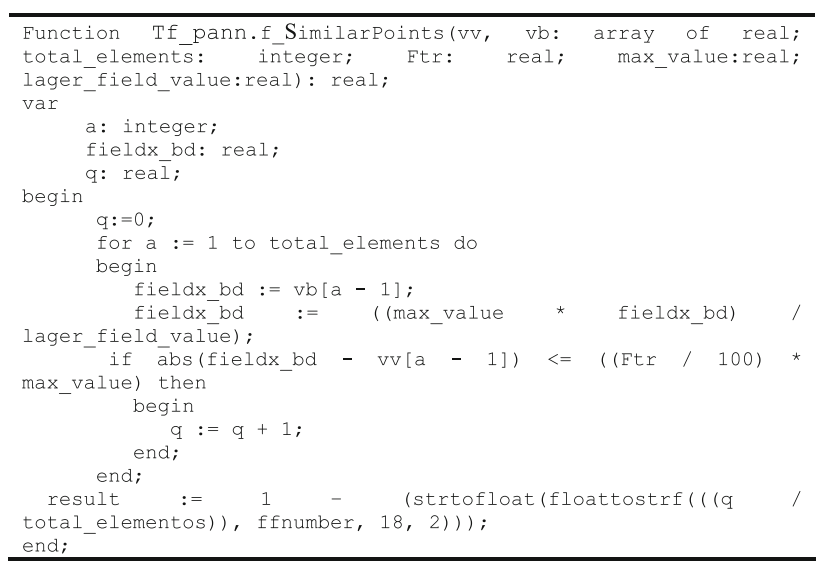

\subsection{Expert system 2: checking similar points}

The aim of the expert system 2 is to compare the waves and analyze their differences regarding to similar points.

When we analyze the similar points, it means that we are analyzing how one approaches the other point.

It is worth remembering that, because it is biological signal, we should not work with absolute quantification due to the variability characteristic of this type of signal. Therefore, one should always take into consideration a tolerance factor.

A sample of checking similar points function implementation using Object Pascal is shown in Table 9.

$\mathrm{Se}_{2}=\frac{\sum_{j=1}^{n}\left(x_{j}\right)}{n}$,

where $n$ is the total number of elements, $x$ is the element of the current position, $\mathrm{a} j$ is the current position, $\mathrm{Se}_{2}$ is the value resulting from the calculation.

\subsection{Expert system 3: checking different points}

The aim of the expert system 3 is to compare the waves and analyze their differences regarding of different points.

When we analyze the different points, it means that we are analyzing how a point more distant from each other, so the factor of tolerance should also be considered.

A sample of checking different points function implementation using Object Pascal is shown in Table 10.

$\mathrm{Se}_{3}=1-\left(\frac{\sum_{j=1}^{n}\left(\frac{\left|x_{j}-y_{j}\right|}{a}\right)}{n}\right)$,

where $n$ is the total number of elements, $a$ is the maximum amount allowed, $j$ is the current position, $x$ is the value of 
Table 10 Checking different points function implementation

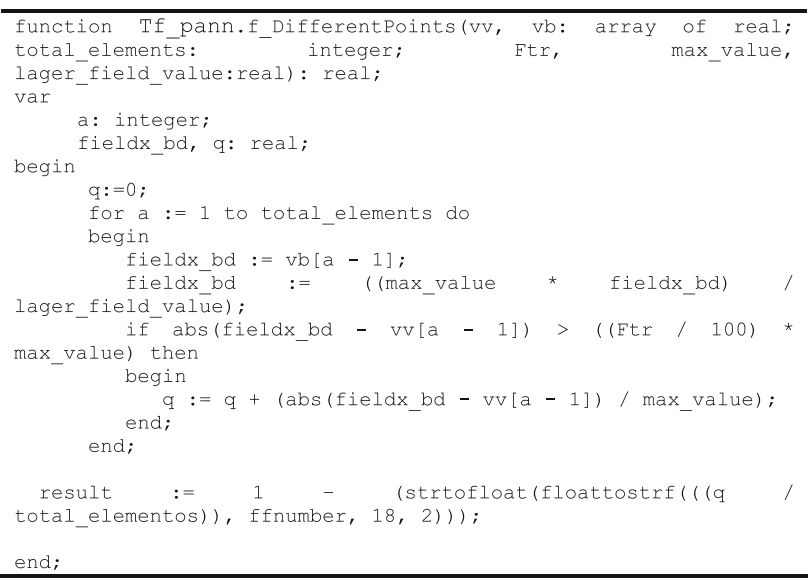

Table 11 Contingency table

\begin{tabular}{lcccccr}
\hline \multicolumn{5}{c}{ Visual analysis } & & \\
\cline { 2 - 7 } & Delta & Theta & Alpha & Beta & Unrecognized & Total \\
\hline PANN Analysis & & & & & \\
Delta & 31 & 3 & 0 & 0 & 0 & 34 \\
Theta & 15 & 88 & 1 & 1 & 0 & 105 \\
Alpha & 0 & 5 & 22 & 0 & 0 & 27 \\
Beta & 0 & 0 & 1 & 3 & 0 & 10 \\
N/D & 7 & 2 & 1 & 0 & 0 & 180 \\
Total & 53 & 98 & 25 & 4 & 0 & \\
\hline
\end{tabular}

Index kappa $=0.80$

wave $1, y$ is the value of wave $2, \mathrm{Se}_{3}$ is the value resulting from the calculation.

\section{Experimental procedures: differentiating frequency bands}

In our work we have studied two types of waves, specifically delta and theta waves band, where the size of frequency established clinically ranges (Fig. 1).

Seven examinations of different EEG were analyzed, being two examinations belonging to adults without any learning disturbance and five examinations belonging to children with learning disturbance $[5,6,13]$.

Each analysis was divided into three rehearsals; each rehearsal consisted of $10 \mathrm{~s}$ of the analyzed, free from visual analysis of spikes and artifacts regarding the channels T3 and T4.

In the first battery of tests, a wave recognition filter belonging to the delta band was considered. In the second one, a wave recognition filter belonging to the theta band was considered. In the third one, none of the filters were considered for recognition (Tables 11, 12, 13, 14, 15, 16).
Table 12 Statistical results—sensitivity and specificity: delta waves

\begin{tabular}{rlrr}
\hline & \multicolumn{2}{l}{ Visual analysis } & \\
\cline { 2 - 4 } & Delta & Not delta & Total \\
\hline PANN & & & \\
True & 31 & 124 & 155 \\
False & 22 & 3 & 25 \\
Total & 53 & 127 & 180 \\
\hline
\end{tabular}

Sensitivity $=58 \%$; specificity $=97 \%$

Table 13 Statistical results—sensitivity and specificity: theta waves

\begin{tabular}{|c|c|c|c|}
\hline & \multicolumn{3}{|c|}{ Visual analysis } \\
\hline & Theta & Not theta & Total \\
\hline \multicolumn{4}{|l|}{ PANN } \\
\hline True & 88 & 65 & 153 \\
\hline False & 10 & 17 & 27 \\
\hline Total & 98 & 82 & 180 \\
\hline \multicolumn{4}{|c|}{ Sensitivity $=89 \%$; specificity $=79 \%$} \\
\hline \multicolumn{4}{|c|}{ Table 14 Statistical results—-sensitivity and specificity: alpha waves } \\
\hline & \multicolumn{3}{|c|}{ Visual analysis } \\
\hline & Alpha & Not alpha & Total \\
\hline \multicolumn{4}{|l|}{ PANN } \\
\hline True & 22 & 150 & 172 \\
\hline False & 3 & 5 & 8 \\
\hline Total & 25 & 155 & 180 \\
\hline
\end{tabular}

Sensitivity $=88 \%$; specificity $=96 \%$

Table 15 Statistical results—sensitivity and specificity: beta waves

\begin{tabular}{rlrr}
\hline & \multicolumn{2}{l}{ Visual analysis } & \\
\cline { 2 - 4 } & Beta & Not beta & Total \\
\hline PANN & & & \\
True & 3 & 175 & 178 \\
False & 1 & 1 & 2 \\
Total & 4 & 176 & 180 \\
\hline
\end{tabular}

Sensitivity $=75 \%$; specificity $=99 \%$

Table 16 Statistical results-sensitivity and specificity: unrecognized waves

\begin{tabular}{rlrr}
\hline & \multicolumn{2}{l}{ Visual analysis } & \\
\cline { 2 - 4 } & Unrecognized & Recognized & Total \\
\hline PANN & & & \\
True & 0 & 170 & 170 \\
False & 0 & 180 & 10 \\
Total & 0 & & 180 \\
\hline
\end{tabular}

Sensitivity $=100 \%$; specificity $=94 \%$ 
Table 17 Lattice for decision-making (Fig. 9) used in diagnostic analysis used after making PANN analysis (Fig. 10)

Characterization of the lattice

\begin{tabular}{cc}
\hline Area 1 & $G_{\mathrm{ce}} \leq 0.1999$ and $G_{\mathrm{ce}} \geq 0.5600$ and $\left|G_{\text {un }}\right|<0.3999$ \\
& and $\left|G_{\mathrm{un}}\right| \geq 0.4501$ \\
Area 2 & $0.2799<G_{\mathrm{ce}}<0.5600$ and $0.3099 \leq\left|G_{\text {un }}\right|<0.3999$ \\
& and $\mathrm{Fe}<0.5000$ \\
Area 3 & $0.1999<G_{\mathrm{ce}}<0.5600$ and $0.3999 \leq\left|G_{\text {un }}\right|<0.4501$ \\
& and Fe $>0.5000$ \\
Area 4 & $G_{\mathrm{ce}}>0.7999$ and $\left|G_{\text {un }}\right|<0.2000$
\end{tabular}

$\mathrm{Ce}$ contrary evidence, $\mathrm{Fe}$ favorable evidence, $G_{\mathrm{ce}}$ certainty degree, $G_{\mathrm{un}}$ uncertainty degree

\section{Experimental procedures: applying in Alzheimer disease}

It is known that the visual analysis of EEG patterns may be useful in aiding the diagnosis of AD and indicated in some clinical protocols for diagnosing the disease $[14,15]$. The most common findings on visual analysis of EEG patterns are slowing of brain electrical activity based on predominance of delta and theta rhythms and decrease or absence of alpha rhythm. However, these findings are more common and evident in patients in moderate or advanced stages of disease $[8,16,17]$.

In this study we have 67 analyzed EEG records, 34 normal and 33 probable AD ( $p$ value $=0.8496$ ) during the awake state at rest.

All tests were subjected to morphological analysis methodology for measuring the concentration of waves. Later this information is submitted to a PANN unit responsible for assessing the data and arriving at a classification of the examination in normal or probable AD (Table 17; Fig. 9).

\subsection{Expert system 1: detecting the diminishing average frequency level}

The aim of the expert system 1 is to verify the average frequency level of alpha band waves and compare them with a fixed external parameter wave.

Such external parameter can be, for instance, the average frequency of a population or the average frequency of the last examination of the patient. This system also generates two outputs: favorable evidence $\mu$ normalized values ranging from 0 (corresponds to $100 \%$-or greater frequency loss) to 1 (which corresponds to $0 \%$ of frequency loss) and contrary evidence $\lambda$ (Eq. 6.1).

The average frequency of population pattern used in this work is $10 \mathrm{~Hz}$.

$\lambda=1-\mu$

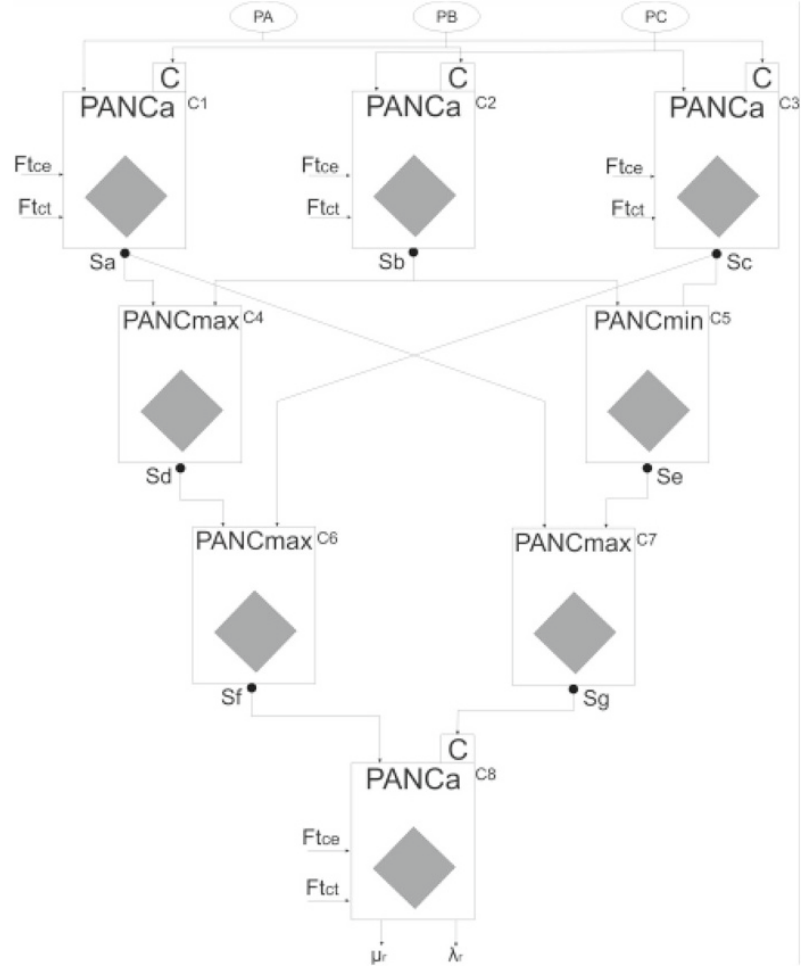

Fig. 9 The architecture for diagnosis analysis

\subsection{Expert system 2: high-frequency band concentration}

The role of the expert system 2 is to analyze alpha band concentration. For this, we consider the quotient of the sum of fast alpha and beta waves over slow delta and theta waves (Eq. 6.2) as first output value. For the second output value (contrary evidence $\lambda$ ) is used Eq. 6.1.

$\mu=\left(\frac{(A+B)}{(D+T)}\right)$

where $A$ is the alpha band concentration; $B$ is the beta band concentration, $D$ is the delta band concentration; $T$ is the theta band concentration; and $\mu$ is the value resulting from the calculation.

\subsection{Expert system 3: low frequency band concentration}

The role of the expert system 3 is to analyze theta band concentration. For this, we consider the quotient of the sum of slow delta and theta waves over fast alpha and beta waves (Eq. 6.3) as first output value. For the second output value (contrary evidence $\lambda$ ) is used Eq. 6.1.

$\mu=\left(\frac{(D+T)}{(A+B)}\right)$ 


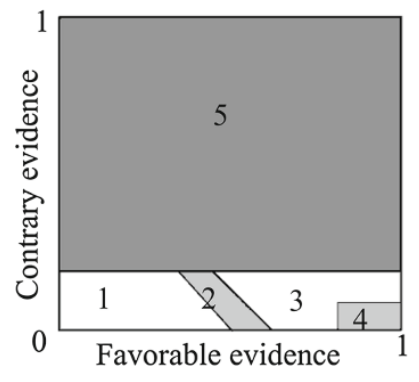

Fig. 10 Lattice for decision-making used in diagnostic analysis (Fig. 9). Area 1 state logical false (AD likely below average population), area 2 state logical Quasi-true (AD likely than average population); area 3 state logical Quasi-false (normal below average population); area 4 state logical true (normal above average population); area 5 logical state of uncertainty (not used in the study area)

where $A$ is the alpha band concentration; $B$ is the beta band concentration. $D$ is the delta band concentration; and $T$ is the theta band concentration. $\mu$ is the value resulting from the calculation.

\subsection{Results}

See Table 18.

\section{Experimental procedures: applying in attention-deficit/hyperactivity disorder (ADHD)}

A similar architecture using PANN was built to study some cases in ADHD. Recent researches reveal that $10 \%$ of the world population in school age suffer of learning and/or behavioral disorders caused by neurological problems, such as ADHD, dyslexia, and dyscalculia, with predictable consequences in those students' insufficient performance in the school [2-6,13].

Concisely, a child without intellectual lowering is characterized as bearer of ADHD when it presents signs of

- Inattention: difficulty in maintaining attention in tasks or games; the child seems not to hear what is spoken; difficulty in organizing tasks or activities; the child loses

Table 18 Diagnosis: normal $\times$ probable AD patients

\begin{tabular}{|c|c|c|c|}
\hline & \multicolumn{3}{|l|}{ Gold standard } \\
\hline & AD patient $(\%)$ & Normal patient (\%) & Total (\%) \\
\hline \multicolumn{4}{|l|}{ PANN } \\
\hline AD patient & 35.82 & 14.93 & 50.75 \\
\hline Normal patient & 8.96 & 40.30 & 49.25 \\
\hline Total & 44.78 & 55.22 & 100.00 \\
\hline
\end{tabular}

Sensitivity $=80 \%$; specificity $=73$; index of coincidence (kappa) $76 \%$ things; the child becomes distracted with any incentive, etc.

- Hyperactivity: frequently the child leaves the class room; the child is always inconveniencing friends; the child runs and climbs in trees, pieces of furniture, etc; the child speaks a lot, etc.

- Impulsiveness: the child interrupts the activities of colleagues; the child does not wait his time; aggressiveness crises, etc.

- Dyslexia: the child begins to present difficulties to recognize letters or to read them and to write them although the child has not a disturbed intelligence, that is, a normal IQ;

- Dyscalculia: the child presents difficulties to recognize amounts or numbers and/or to figure out arithmetic calculations.

A child can present any combination among the disturbances above. All those disturbances have their origin in a cerebral dysfunction that can have multiple causes, many times showing a hereditary tendency.

Since from the first discoveries, those disturbances have been associated with cortical diffuse lesions and/or more specific, temporal-parietal areas lesions in the case of dyslexia and dyscalculia [2,5,13].

The disturbances of ADHD disorder seem to be associated with an alteration of the dopaminergic system, that is, it is involved with mechanisms of attention and they seem to involve a frontal-lobe dysfunction and basal ganglia areas $[3,13]$.

EEG alterations seem to be associated with those disturbances. Thus, some authors have proposed that there is an increase of the delta activity in EEG in those tasks that demand a larger attention to the internal processes.

Other authors [1] have described alterations of the delta activity in dyslexia and dyscalculia children sufferers. Klimesch [18] has proposed that a phase of the EEG component would be associated with the action of the memory work. More recently, Kwak [19] has showed delta activity is reduced in occipital areas, but not in frontals, when dyslexic children were compared with normal ones.

In this way, the study of the delta and theta bands becomes important in the context of the analysis of learning disturbances.

So, in this paper we have studied two types of waves, specifically delta and theta wave bands, where the size of frequency established clinically ranges $1.0-3.5$ and 4.0-7.5 $\mathrm{Hz}$, respectively.

Seven exams of different EEG were analyzed, being two exams belonging to adults without any learning disturbance and five exams belonging to children with learning disturbances (exams and respective diagnoses given by 
ENSCER - Teaching the Brain, EINA—Studies in Natural Intelligence and Artificial Ltda).

Each analysis was divided into three rehearsals, and each rehearsal consisted of $10 \mathrm{~s}$ of the analyzed, free from visual analysis of spikes and artifacts regarding the channels $\mathrm{T} 3$ and T4. In the first battery of tests, a delta recognition filter wave was considered. For second battery of tests, a theta recognition wave was considered. For the third battery of tests, none of the filters were considered for recognition, i.e., the system worked freely for any wave type recognition. The total number of exams is 180 (Tables 19, 20, 21, 22, 23, 24).

\section{Conclusions}

We believe that a process of the examination analysis using a PANN attached to EEG findings, such as relations between

Table 19 Contingency table

\begin{tabular}{lcccccr}
\hline \multicolumn{7}{c}{ Visual analysis } \\
\cline { 2 - 7 } & Delta & Theta & Alpha & Beta & Unrecognized & Total \\
\hline PANN analysis & & & & & \\
Delta & 31 & 3 & 0 & 0 & 0 & 34 \\
Theta & 15 & 88 & 1 & 1 & 0 & 105 \\
Alpha & 0 & 5 & 22 & 0 & 0 & 27 \\
Beta & 0 & 0 & 1 & 3 & 0 & 4 \\
N/D & 7 & 2 & 1 & 0 & 0 & 0 \\
Total & 53 & 98 & 25 & 4 & 0 & 180 \\
\hline
\end{tabular}

Index kappa $=0.80$

Table 20 Statistical results—sensitivity and specificity: delta waves

\begin{tabular}{llrr}
\hline & \multicolumn{2}{l}{ Visual analysis } & \\
\cline { 2 - 4 } & Delta & Not delta & Total \\
\hline PANN analysis & & & \\
True & 31 & 124 & 155 \\
False & 22 & 3 & 25 \\
Total & 53 & 127 & 180 \\
\hline
\end{tabular}

Sensitivity $=58 \%$; specificity $=97 \%$

Table 21 Statistical results—-sensitivity and specificity: theta waves

\begin{tabular}{lllr}
\hline & \multicolumn{2}{l}{ Visual analysis } & \\
\cline { 2 - 4 } & Theta & Not theta & Total \\
\hline PANN analysis & & & \\
True & 88 & 65 & 153 \\
False & 10 & 17 & 27 \\
Total & 98 & 82 & 180 \\
\hline
\end{tabular}

Sensitivity $=89 \% ;$ specificity $=79 \%$
Table 22 Statistical results—-sensitivity and specificity: alpha waves

\begin{tabular}{lcrr}
\hline & \multicolumn{2}{l}{ Visual analysis } & \\
\cline { 2 - 4 } & Alpha & Not alpha & Total \\
\hline PANN analysis & & & \\
True & 22 & 150 & 172 \\
False & 3 & 5 & 8 \\
Total & 25 & 155 & 180 \\
\hline
\end{tabular}

Sensitivity $=88 \%$; specificity $=96 \%$

Table 23 Statistical results-sensitivity and specificity: beta waves

\begin{tabular}{llrr}
\hline & \multicolumn{2}{l}{ Visual analysis } \\
\cline { 2 - 4 } & Beta & Not beta & Total \\
\hline PANN analysis & & & \\
True & 3 & 175 & 178 \\
False & 1 & 1 & 2 \\
Total & 4 & 176 & 180 \\
\hline
\end{tabular}

Sensitivity $=75 \%$; specificity $=99 \%$

Table 24 Statistical results—sensitivity and specificity: unrecognized waves

\begin{tabular}{llrr}
\hline & \multicolumn{2}{l}{ Visual analysis } \\
\cline { 2 - 4 } & Unrecognized & Recognized & Total \\
\hline PANN analysis & & & \\
True & 0 & 180 & 180 \\
False & 0 & 0 & 0 \\
Total & 0 & 180 & 180 \\
\hline
\end{tabular}

Sensitivity $=100 \%$; specificity $=100 \%$

frequency bandwidth and inter hemispheric coherences, can create computational methodologies that allow the automation of analysis and diagnosis.

These methodologies could be employed as tools to aid in the diagnosis of diseases such as dyslexia or Alzheimer, provided they have defined electroencephalographic findings.

In the case of Alzheimer's disease, for example, the studies carried out previously have shown satisfactory results [20] (but still far from being a tool to aid clinical) that demonstrated the computational efficiency of the methodology using a simple morphological analysis (only paraconsistent annotated logic $E \tau$ ). These results encouraged us to improve the morphological analysis of the waves and try to apply the method in other diseases besides Alzheimer's disease.

With the process of morphological analysis using the PANN, it becomes possible to quantify the frequency average of the individual without losing its temporal reference. This feature becomes a differential, compared to traditional analysis of quantification of frequencies, such as fast Fourier 
transform, aiming at a future application in real-time analysis, i.e., at the time of acquisition of the EEG exams.

Regarding the specificity, the method showed more reliable results. Taking into account an overall assessment in the sense we take the arithmetic mean of sensitivity $(75.50 \%)$ and specificity $(92.75 \%)$, we find reasonable results that encourage us to seek improvements in this study.

The consideration of morphological analysis of the main brain waves by employing PANN showed be effective, allowing interesting quantitative and qualitative examinations of EEG data. PANN has been applied in other branches: MICR automated recognition [16], computer-aided diagnosis (breast cancer) [17], and many other themes.

Open Access This article is distributed under the terms of the Creative Commons Attribution License which permits any use, distribution, and reproduction in any medium, provided the original author(s) and the source are credited.

\section{References}

1. Niedermeyer, E., da Silva, F.H.L.: Electroencephalography, 5th edn. Lippincott Williams \& Wilkins, Philadelphia (2005)

2. Ansari, D., Karmiloff-Smith, A.: Atypical trajectories of number development: a neuroconstructivist perspective. Trends Cogn. Sci. 12, 511-516 (2002)

3. Blonds, T.A.: Attention-deficit disorders and hyperactivity. In developmental disabilities in infancy and Ramus, F., developmental dyslexia: specific phonological deficit or general sensorimotor dysfunction? Curr. Opin. Neurobiol. 13, 1-7 (2003)

4. Hynd, G.W., Hooper, R., Takahashi, T.: Dyslexia and languagebased disabilities. In: Coffey, C.E., Brumbak, R.A. (eds.) Text Book of Pediatric Neuropsychiatry, pp. 691-718. American Psychiatric Press, Washington, DC (1985)

5. Lindsay, R.L.: Dyscalculia. In: Capute, A.J., Accardo, P.J. (eds.) Developmental Disabilities in Infancy and Childhood, pp. 405415. Paul Brookes Publishing Co, Baltimore (1996)

6. Temple, E.: Brain mechanisms in normal and dyslexic readers. Curr. Opin. Neurobiol. 12, 178-183 (2002)

7. Kwak, Y.T.: Quantitative EEG findings in different stages of Alzheimer's disease. J. Clin. Neurophysiol. 23(5), 456-461 (2006)

8. Duffy, F.H., Albert, M.S., Mcnulty, G., Garvey, A.J.: Age differences in brain electrical activity of healthy subjects. Ann. Neural 16, 430-438 (1984)
9. Nuwer, M.R., Comi, G., Emerson, R., Fuglsang-Frederiksen, J., GuériT, M., Hinrichs, H., Ikeda, A., Luccas, F.J.C., Rappelsberger, P.: IFCN standards for digital recording of clinical EEG. Electroencephalogr. Clin. Neurophysiol. 106, 259-261 (1998)

10. Nitrini, R., Caramelli, P., Bottino, C.M., Damasceno, B.P., Brucki, S.M., Anghinah, R.: Academia Brasileira de Neurologia. Diagnosis of Alzheimer's disease in Brazil: diagnostic criteria and auxiliary tests. Recommendations of the Scientific Department of Cognitive Neurology and Aging of the Brazilian Academy of Neurology. Arq Neuropsiquiatr. 63(3A), 9-713 (2005)

11. Da Silva Filho, J.I., Torres, G.L., Abe, J.M.: Uncertainty Treatment Using Paraconsistent Logic-Introducing Paraconsistent Artificial Neural Networks, vol. 211. IOS Press, Netherlands (2010). ISBN 978-1-60750-557-0. doi:10.3233/978-1-60750-558-7-I

12. Abe, J.M.: Foundations of annotated logics. PhD thesis (in Portuguese) USP, Brazil (1992)

13. Voeller, K.K.S.: Attention-deficit/hyperactivity: neurobiological and clinical aspects of attention and disorders of attention. In: Coffey, C.E., Brumbak, R.A. (eds.) Text Book of Pediatric Neuropsychiatry, pp. 691-718. American Psychiatric Press, Washington, D.C (1998)

14. Claus, J.J., Strijers, R.L.M., Jonkman, E.J., Ongerboer De Visser, B.W., Jonker, C., Walstra, G.J.M., Scheltens, P., Gool, W.A.: The diagnostic value of EEG in mild senile Alzheimer's disease. Clin. Neurophysiol. 18, 15-23 (1999)

15. Crevel, H., Gool, W.A., Walstra, G.J.M.: Early diagnosis of dementia: which tests are indicated? What are their costs. J. Neurol. 246, 73-78 (1999)

16. Souza, S., Abe, J.M., Nakamatsu, K.: MICR Automated Recognition Based on Paraconsistent Artificial Neural Networks, Procedia Computer Science, vol. 22, pp. 170-178. Elsevier, London (2013)

17. Amaral, F.V.: Paraconsistent mammography image attributes classifier in breast cancer diagnosis: based on paraconsistent artificial neural network. PhD thesis (in Portuguese) UNIP, Brazil (2013)

18. Klimeshc, W.: EEG alpha and theta oscillations reflect cognitive and memory performance: a review and analysis. Brain Res. Ver. 29, 169-195 (1999)

19. Klimesch, W., Doppelmayr, H., Wimmer, J., Schwaiger, D., Rôhm, D., Bruber, W., Hutzler, F.: Theta band power changes in normal and dyslexic children. Clin. Neurophysiol. 113, 1174-1185 (2001)

20. Lopes, H.F.S.: Aplicação de redes neurais artificiais paraconsistentes como método de auxílio no diagnóstico da doença de Alzheimer. MSc Dissertation (in Portuguese), Faculdade de Medicina-USP, São Paulo (2009) 